Eurostudia

\title{
La cloche sonne, le temps passe
}

Sophie Coulombe

Volume 12, numéro 1, 2017

\section{Feuilleton}

URI : https://id.erudit.org/iderudit/1041663ar

DOI : https://doi.org/10.7202/1041663ar

Aller au sommaire du numéro

Éditeur(s)

Le Centre canadien d'études allemandes et européennes

ISSN

1718-8946 (numérique)

Découvrir la revue

Citer ce document

Coulombe, S. (2017). La cloche sonne, le temps passe. Eurostudia, 12(1), 75-81.

https://doi.org/10.7202/1041663ar

Tous droits réservés ( Le Centre canadien d'études allemandes et européennes, 2017
Ce document est protégé par la loi sur le droit d'auteur. L'utilisation des services d’Érudit (y compris la reproduction) est assujettie à sa politique d'utilisation que vous pouvez consulter en ligne.

https://apropos.erudit.org/fr/usagers/politique-dutilisation/
Cet article est diffusé et préservé par Érudit.

Érudit est un consortium interuniversitaire sans but lucratif composé de l’Université de Montréal, l’Université Laval et l'Université du Québec à Montréal. Il a pour mission la promotion et la valorisation de la recherche. https://www.erudit.org/fr/ 


\section{Sophie Coulombe \\ Université de Montréal}

Les institutions [totales] sont donc incompatibles avec cette structure de base de notre société qu'est le rapport travail-salaire.

Erving Goffman, Asiles, 1968, p. 53

Ce matin-là, il y avait une grève des employées et employés de l'administration. Le piquetage étant interdit sur le terrain de la prison, les grévistes brandissaient leurs pancartes devant le bureau de poste, de l'autre côté de la rue... J'ai alors remarqué que je n'avais pratiquement jamais croisé de membres du personnel administratif à l'intérieur des murs. Les agentes et agents des services correctionnels étaient aussi en processus de négociation avec leur employeur. N'ayant pas le droit de grève, ils affichaient brassards, cocardes ou casquettes en guise de moyens de pression pendant l'exercice de leurs fonctions. En prison, les femmes aussi travaillent, du moins, celles qui en ont la "chance », puisqu'il n'y a pas plus qu'une cinquantaine de postes pour plus d'une centaine de femmes détenues.

J'arrive dans le hall d'entrée et l'agente à l'accueil ne trouve pas mon nom sur le registre des visites autorisées aujourd'hui. À ce moment, cela fait déjà trois mois que je viens à la prison presque chaque semaine. Elle appelle l'agent de programmation : «Ça te dit-tu quelque chose, Sophie Coulombe ? " Je n'entends pas sa réponse, mais celle de l'agente devant moi : «ben, c'est pas ma faute, moi je n'ai rien ici ». La faute des papiers perdus passe d'une classe d'employées et employés à une autre, l'agent de programmation accusant le personnel de l'accueil et vice versa. Je signe finalement le registre de présence, l'agente me donne un cadenas et une cocarde avec la mention «Invité ». Je range mes effets dans le casier : cellulaire, sac, cigarettes (je n'ai droit qu'à mon carnet de notes et mon crayon). N'ayant pas de poches, j'accroche la clé du casier à la cocarde et j'ai une petite pensée pour la petite clé souvent accrochée 
au cou ou au poignet des femmes ${ }^{1}$. Assise face aux casiers, $j^{\prime}$ attends sur un banc. Quelques minutes passent avant qu'on vienne à ma rencontre pour me conduire au bureau de Raymond, un membre du personnel affilié à la Commission scolaire de Montréal ${ }^{2}$. J'avais croisé Raymond lors d'une activité à la chapelle de la prison et il m'avait alors suggéré de venir passer une journée sur les différents plateaux de travail de la prison. C'est donc à sa suggestion que j'ai passé la matinée à la buanderie et l'après-midi à la friperie.

Lorsque j'arrive à son bureau, Raymond me sourit, une boîte de beignes à la main. Nous redescendons ensemble dans le hall d'entrée afin que je puisse reprendre mon manteau, car nous devrons marcher à l'extérieur pour nous rendre à la buanderie. En marchant au sous-sol de la prison pour aller rejoindre la porte menant à l'extérieur, Raymond m'avise qu'il fera une petite visite aux femmes travaillant à l'entretien ménager. Elles ne sont que deux, leurs principales tâches consistent à nettoyer les planchers des corridors de la prison ainsi que les différentes salles communes. C'est vendredi et Raymond a l'habitude d'acheter des beignes pour les travailleuses, qu'il nomme affectueusement les «travaillantes », et il voulait s'assurer d'en offrir à cellesci. Après avoir discuté un peu avec les deux femmes, nous continuons notre chemin vers la buanderie. Il mentionne au passage que la distribution de beignes est une pratique critiquée par le personnel correctionnel et qu'il a dû insister afin qu'elle soit tolérée. À la prison, on ne peut pas apporter des choses de l'extérieur et les distribuer aux femmes, à part pour quelques exceptions comme les vêtements de la friperie.

Nous passons les portes de la buanderie où les femmes sont déjà au travail depuis $8 \mathrm{~h} 30$. Il s'agit d'un véritable milieu de travail industriel, la prison sous-traitant des contrats de lavage, avec les hôpitaux notamment. Le bruit des machines est saisissant. Les femmes qui y travaillent sont en équipe et sont attitrées à différents postes : le triage, le lavage, le séchage, le pliage et le repassage. L'horaire de travail est de $8 \mathrm{~h} 30$ à $11 \mathrm{~h}$ et de $12 \mathrm{~h}$ à $14 \mathrm{~h}$, et prévoit une pause-café de quinze minutes. Le salaire en est de 3,69\$ de

\footnotetext{
1 Voir Thériault et Coulombe (2017b).

2 Les enseignantes et enseignants des cours de français, de mathématique, d'anglais et de francisation, de même que le personnel en intégration socioprofessionnelle de la prison, sont affilié.es à la Commission scolaire de Montréal.
} 
l'heure ${ }^{3}$. Malgré les bas salaires, les postes à la buanderie sont convoités et il y a une liste d'attente. La plupart des femmes veulent travailler, non seulement pour amasser de l'argent pour la cantine, mais aussi parce que cela permet de faire «son temps » sans trop s'ennuyer.

Raymond et moi sommes debout, immobiles, proches des cabinets de toilette. Raymond parle beaucoup. Les membres du personnel ont l'habitude de se raconter : «Moi, je suis de cet avis »; "Moi, je préfère faire comme ça »; «Moi, je suis en désaccord avec...»; «Moi, je crois qu'il faut faire ainsi ». J'essaie d'écouter Raymond tout en observant les femmes au travail. Quelques bouts de discours sur la réhabilitation et la responsabilisation des femmes écorchent mes oreilles. Raymond affirme que, malgré tout, le crime est souvent une question de choix et les femmes doivent être responsabilisées. Il enchaîne sur les conflits de travail et c'est avec agacement qu'il affirme ne pas avoir le choix de se positionner, dans la majorité des cas, du côté du personnel. Il n'a pas le choix, ça semble catégorique, il risquerait trop. Ce n'est pas la première fois non plus que je perçois cette ambiguïté chez les membres du personnel : particulièrement entre les membres du personnel non correctionnel et les agentes et agents. Raymond s'est aussi mis à parler de ses projets de retraite : il rêve de la campagne, de s'éloigner de la civilisation. Dans sa voix, il y a quelque chose qui m'échappe, une amertume peut-être. Peut-être se sent-il coincé ; j'aurais pu lui demander, mais je m'affaire à observer les femmes et le laisse dans ses réflexions. Les femmes travaillent tout en discutant. Elles enchaînent de manière minutieuse les pliages de jaquettes d'hôpital, de draps et de serviettes. Il y a tellement de bruit que j'entends à peine ce qu'elles se disent. Je m'installe à une table et les femmes me montrent fièrement la technique de pliage de chaque vêtement, elles insistent sur la précision, tous les coins pliés doivent être parfaitement alignés. Je plie en silence, les femmes discutent à voix basse, je les entends s'interpeller souvent par des petits noms : «babe », « chérie », « ma belle ».

C'est l'heure de la pause-café. Visiblement heureuses d'avoir aujourd'hui un beigne pour collation, les femmes remercient Raymond.

\footnotetext{
${ }^{3}$ La rémunération d'une personne incarcérée pour le travail exécuté en prison est fixée à $35 \%$ du salaire minimum au Québec. Elle est donc passée à 3,93\$ de l’heure le 1“ mai 2017. (Voir la page sur le «Programme d'activités en milieu carcéral » du ministère de la Sécurité publique du Québec, au www.securitepublique.gouv.qc.ca / services-correctionnels / reinsertion-sociale/ programmes-servicesactivites / activites-milieu-carceral.html [consultée le 4 septembre 2017]).
} 
Comme dans beaucoup de milieux de travail, les femmes se rassemblent autour de la machine à café. Nous enchaînons les sujets de discussion : quel est notre beigne préféré ? L'hiver approche. Que mange-t-on pour dîner ? Que feront-elles à leur sortie de prison ? Rares sont les moments de silence. Nous discutons aussi d'emplois : l'une des femmes, dans la jeune vingtaine, parle fièrement de ses talents musicaux et se tarde de retrouver son ordinateur pour créer à nouveau de la musique. La femme à sa droite réagit et lui répond la voix un peu cassée : «Ouin, tu as de la chance, moi j'ai tout tout tout perdu depuis mon incarcération. " Les femmes témoignent un court moment d'empathie, puis repartent la discussion sur un autre sujet. À la différence de certaines conversations de bureau qui peuvent rester très superficielles, les discussions entre les femmes en prison sont souvent déconcertantes: on y retrouve une sorte d'authenticité et de spontanéité faisant que des sujets aussi légers que la saveur préférée de la crème glacée ou le film que l'on a écouté hier peuvent s'entremêler à des sujets difficiles tels que la perte de la garde d'un enfant ou la violence d'un mari.

La cloche sonne, c'est l'heure de reprendre le travail. C'est parti pour une heure et demie de pliage. Sur la table, les jaquettes et les serviettes s'empilent à une vitesse impressionnante. Une des femmes fredonne et, tranquillement, d'autres l'accompagnent. Raymond et une femme discutent. Comme il est de l'autre côté de la table, je ne les entends pas très bien, mais en les observant - j'ai l'impression que les femmes voient en lui un allié.

Il est 11 h, c'est l'heure du dîner. Les femmes s'entassent devant la porte de sortie. En passant devant le bureau de l'agente de supervision, je remarque au mur, à l'intérieur, des outils ayant tous en commun la particularité d'être coupants: ciseaux, couteaux à lame rétractable, cisailles, coupe-boulons, pinces à couper. Toutes ces lames mises à l'abri rappellent le lieu où nous nous trouvons. Dehors, nous marchons en file sur un petit chemin asphalté. À la rentrée dans le bâtiment principal, Raymond fait le décompte et, dans un petit moment d'inattention, il m'inclut dans son total. Les femmes rient, font quelques blagues à Raymond et me disent de faire attention pour ne pas rester « pognée » ici.

Mis à part une femme qui attend pour parler à Raymond, les autres se dirigent vers leur secteur respectif où elles prennent leurs repas. Seul le personnel mange à la cafétéria, les femmes mangeant dans l'aire commune de 
leur secteur. J'attends Raymond dans le couloir. Dans le secteur d'à côté, les femmes se servent à manger. Ce sont elles qui font le service et apportent les charriots remplis de plateaux de repas. Ensuite, chaque table du secteur est appelée à venir chercher son repas. Les femmes du secteur me regardent un peu de travers ; je me rends compte que je les observe derrière la vitre... Je souris bêtement et détourne le regard. Nous nous dirigeons finalement vers la cafétéria des employées et employés pour le dîner. Je suis assise avec Raymond et d'autres membres du personnel. Nous discutons recettes, décoration intérieure et films. L'amatrice de cinéma que je suis se joint à la conversation un instant, mais les uniformes qui m'entourent me ramènent rapidement à la réalité, à celles qui mangent de l'autre côté de ces murs, à celles qui sont enfermées. Et, pour ajouter à l'ironie de la situation, nous discutons du film Shawshank Redemption...

Raymond et moi avions convenu que je passerais l'après-midi à la friperie. Le repas terminé, Raymond et moi montons donc à l'étage pour ouvrir la petite pièce où les vêtements sont entreposés. Raymond appelle les secteurs des deux femmes qui travaillent à la friperie afin d'aviser qu'elles peuvent nous rejoindre. La friperie est une toute petite pièce de la superficie d'une chambre à coucher, avec un petit local attenant où se trouvent des vêtements et des accessoires entassés dans des sacs. Tous les murs du local principal sont couverts d'étagères grimpant jusqu'au plafond. Au centre, il y a deux paniers de triage en métal et une table. Le travail consiste à trier les vêtements : «ce qui passe » et «ce qui ne passe pas ». Les camisoles à bretelles «spaghetti», les vêtements trop transparents ou trop «suggestifs", les vêtements à capuchon et, bien évidemment, les vêtements trop usés ou tachés ne «passent pas ». Ensuite, les femmes doivent écheler les étagères pour poser le linge dessus ; pour l'instant, il est placé par tailles et types: chandails chauds, t-shirts, pantalons, jeans, pyjamas.

Les femmes arrivent. Lyne, début cinquantaine, termine son séjour la semaine prochaine et Sandra, début trentaine, vient de commencer son travail à la friperie. Je leur serre la main et me présente. Lyne me lance un bonjour un peu sec. Je lui mentionne que nous nous sommes croisées au Café pasto la

4 Chaque aire commune est équipée de tables, de chaises, d'armoires, d'un micro-onde et d'un réfrigérateur. Les femmes appellent souvent cette zone la «cuisine». On y trouve également un téléviseur. Un seul secteur de la prison n'est pas équipé ainsi, celui destiné aux personnes souffrant de troubles de santé mentale. Il est à noter que certaines femmes préfèrent manger dans leur cellule. 
semaine dernière et elle me répond tout aussi sèchement: "Peut-être, je ne sais pas, on est tellement ici... » Sa réponse me fait sourire ; je lui précise que je suis l'étudiante chercheuse qui travaille sur le quotidien en prison et elle éclate soudainement de rire : «Je pensais que tu étais une des filles. » Son attitude à mon égard change du tout au tout et elle semble heureuse de m'expliquer le fonctionnement de la friperie.

Le local adjacent à la friperie est situé juste à côté d'un étroit passage qui mène au sous-sol, aux salles de classe. Des femmes reviennent tout juste de l'école. Une d'entre elles - elle me semble très jeune et a une ecchymose à l'œil droit — s'arrête et explique à Raymond qu'elle s'est fait «briser » son linge dans son secteur. Après une hésitation marquée, Raymond accepte qu'elle prenne de nouveaux vêtements - je me demande si ma présence a eu une influence sur sa décision. La jeune femme, en se faisant aider par Lyne et Sandra, choisit quelques morceaux, insiste pour en prendre plus, n'obtient pas l'approbation, remercie Raymond et les deux femmes pour ensuite repartir. Raymond téléphone au secteur de la jeune femme afin d'aviser qu'elle a été autorisée à prendre des vêtements. Lors de cet appel téléphonique, Lyne m'explique l'histoire des vêtements endommagés et de l'ecchymose. Elle me raconte que la jeune femme serait kleptomane et déroberait «tout» dans le secteur, «même le pain!», me précise Sandra. Des femmes ont voulu lui donner une leçon et... La violence entre les femmes est omniprésente en prison. Les femmes m'ont souvent dit qu'il était quand même rare qu'elle soit exprimée par des coups, mais cela arrive. Lyne ajoute que la jeune femme à l'ecchymose le méritait ; à ses mots, Raymond fronce les sourcils. Elle se reprend aussitôt en disant que c'est faux, que personne ne le mérite et, qu'en plus, elle est malade.

Le travail reprend son cours. À tour de rôle, des femmes qui avaient pris rendez-vous viennent sélectionner quelques morceaux de vêtements et les deux travailleuses les conseillent avec sérieux. La plupart des femmes insistent pour avoir quelques morceaux de plus, mais le règlement n'autorise la possession que de 34 articles et les échanges ne sont possibles que deux fois par années. Il est bientôt $14 \mathrm{~h}$ et la friperie va fermer. Pour ces femmes, tant celles de la buanderie que de la friperie, mais aussi pour celles qui travaillent à l'entretien ménager ou à la cuisine et celles qui vont à l'école, le congé de fin

\footnotetext{
${ }^{5}$ Parmi ces 34 articles, les femmes ont droit à quatre hauts (chandails) et quatre bas (pantalons).
} 
de semaine arrive. Pour la plupart d'entre nous à l'extérieur de la prison, la fin de semaine est le moment privilégié pour reprendre son souffle et faire des activités récréatives. Pour les femmes en prison, il s'agit plutôt d'un moment de désœuvrement. Au fond, elles rejoignent celles qui n'ont pas la «chance » de travailler ou d'aller à l'école, faute de place, et qui, la semaine durant, cherchent par tous les moyens à passer le temps.

À ma sortie, les grévistes n'étaient plus là. Cela m'a fait penser qu'il est certain que les revendications des femmes pour avoir plus d'emplois, plus d'activités et d'occupations en prison resteront pour leur part non entendues. En effet, il est peu probable qu'une grève ou d'autres moyens de pression soient tolérés, ni par l'institution, ni par celles et ceux-là mêmes qui, ce matin, revendiquaient de meilleures conditions de travail.

\section{Bibliographie}

Kracauer, Siegfried. (2008 [1963]) L'ornement de la masse : Essais sur la modernité weimarienne. Paris, La Découverte.

_. (1995 [1964]) Rue de Berlin et d'ailleurs. Paris, Gallimard.

—_. (2012 [1929/1930]) Les Employés. Paris, Les Belles Lettres.

Goffman, Erving. (1968) Asiles. Études sur la condition sociale des malades mentaux. Lonrai, Les Éditions de minuit.

Thériault, Barbara (avec la collaboration de Sophie Coulombe). (2017) «Die den Rosenkranz tragen. Religiöse Formen in einem Frauengefängnis », in Architektur \& Artefakte. Zur materialen Seite des Religiösen. Karstein, Uta et Thomas Schmidt-Lux (éds), Wiesbaden, VS Verlag für Sozialwissenschaften. 